\title{
LIBII NUOVI
}

\section{THERMODYNAMIOUE ET CHIMIE,}

par P. DUHEM.

(Paris, A. Hermann, 1902 ).

Per facilitare sempre più ai cultori della chimica l'intelligenza delle nozioni fondamentali della termodinamica, il Juhem ha scritto un corso di lezioni, nelle quali in forma chiara e semplice espone tutto ciỏ che può servire di guida sia nello studio del grandissimo numero di fatti chimici già noti, sia nelle nuove ricerche, mostrando quali sono, in ciascun tipo di reazioni, le condizioni variabili di cui si puó disporre e le circostanze essenziali che ciascuna volta debbono esser deterninate.

Perche le regole della termodinamica siano applicate a dovere, occorre conoscere da quali principi esse derivano, $\theta$ in quali condizioni son valide. Perciò il Duhem ha consacrato le prime cinque lezioni all' esposizione dei fondamenti della statica e della dinamica chimica. In questa trattazione dei principi della termodinamica si fa uso solamente delle nozioni di algebra elementare; per dimostrazioni piu complete, nelle quali occorre il calcolo superjore, serve l' altra opera del Duhem sulla Meccanica chimica fondata sulla termodinamica (clir. N. Cim. (4), 6, p. 227). Nel libro di cui ora ci occupiamo è stato dato largo posto alle applicazioni più recenti della termodinamica alla chimica, specialmente alla regola delle fasi, che è una delle piu feconde e preziose della chimica moderna. Uno studio assai esteso è destinato anche ai cristalli misti, ai conglomerati eutectici e agli stati indifferenti dei miscugli doppi, le cui proprietà sono assai fallaci; e nulla è stato trascurato per mettere lo sperimentatore in guardia contro quei simulatori dell' analisi chimica.

Ciascuna regola è illustrata da numerosi esempi, i quali non solo ne fanno apprezzare l'importanza, ma fanno anche conoscere quali precauzioni occorrano nell' adoperarle. 
Insieme alle lezioni del van't Hoff, anche queste del Duhem mostrano sempre più quanto sia grande il sussidio, che lo studio della chimica riceve dalle cognizioni della fisica e della matematica; cognizioni di cui non dovrebbe difettare nessun cultore delle scienze naturali.

\section{A. Stefanini.}

\section{MATHEHATISGHE OPTIK, \\ von J. CLASSEN. \\ (Leipzig, f. J. Gösehen, 19(1)).}

Isa parte dell' ottica che è trattata in questo volume si scosta assai da quella che è esposta negli ordinari manuali. Infatti, nei trattati di ottica, se sono fondati su un qualunque concetto della natura della luce, si suole esporre prevalentemente i problemi della teoria ondulatoria, come l' interferenza, la polarizzazione, la doppia refrazione ecc., mentre le leggi dell' ottica geometrica sono appena accennate. In rersamente, quei trattati che si occupano di ottica geometrica non si occupano della teoria ondulatoria, trascurando i fenomeni di diffrazione, che pure hanno tanta parte nella formazione delle immagini.

Il concetto che ha servito di guida a questo libro porta ad una diversa divisione e limitazione della materia.

Da prima, seguendo il procedimento della Fisica teorica del Voigt, l'A. assume come dati alcuni fatti sperimentali semplici, e ricerca la forma che deve avere una funzione analitica che possa rappresentarli completamente, ed in tal modo ottiene la forma generale dell' equ zione delle onde luminose. In un breve capitolo sono poi svolte alcune relazioni matematiche generali, che vilgono per tale funzione $\theta$ si accenna alla loro analogia con alcune altre della teoria elastica e di quella elettromagnetica. Con ciò è spiegata la possibilità di concepire i fenomeni, rappresentati da quella funzione, tanto nel concetto della elasticita che in quello dell' elettromagnetismo.

Vengono in seguito le applicazioni della rappresentazione matematica all' interferenza, che è esposta nella forma più 
essenziale. Ne vien poi dedotto il principio di Huygens nella sua forma più generale, e la propag zzione rettilinea della luce, di cui sono immediate conseguenze la riflessione e la rifrazione, e le leggi della formazione delle immagini nei fasci astigmatici. Secondo il medesimo concetto vengon poi esposte le relazioni puramente geometriche fra un oggetto e la sua immagine ottica, tanto pei sistemi catottrici che per quelli diottrici.

Gli ultimi capitoli trattano dell' acromasia delle lenti, delle aberrazioni delle immagini, e dei fenomeni di diffrazione.

\section{A. Stefanini.}

\section{L'ASSIMILAZIONE DEL GARBONIO,}

di R. BETIINI.

(R. Giusti, Livorno 1902).

Questo lavoro, pel modo con cui è trattato, puó interessare ad un tempo il botanico, il chimico ed il fisiologo.

L'A. comincia col presentare il fenomeno nelle sue linee generali riferendosi ai concetti moderni della conservazione dell' energia ed al grande ciclo di trasformazioni che avvengono nella meccanica biologica. Imprende quindi a studiare come si presenti l'assimilazione negli organismi inferiori $\theta$ dagli studi di Winogradsky, di Engelmann e di Kohl, deduce importanti conclusioni sull' adattamento e sull' evoluzione degli organismi alle varie specie di energia, notando come nella scala della vita i fenomeni fotosintetici avvengano prima per le radiazioni calorifiche dello spettro oscuro gradualmente passando alle più rapide dello spettro luminoso.

Nel III capitolo l'A. tratta della clorofilla, della formazione sua, degli studi chimici e del sigraificato che acquista la sua presenza nelle piante. La estesa parte bibliografica è discussa con acutezza. Nè mancano alcune esperienze a convalidare l'opinione dell'A. che accetta in massima l'ipotesi di Reinke.

Venendo a trattare dei primi prodotti che si formano nel processo fotosintetico l'A. combatte per vie nuove alcune ipo- 
tesi. Egli introduce l' analisi termochimica delle formule adottate da alcuni scienziati, notando come esse sieno in contradizione col fatto essenziale, pel quale si ammette che il fenomeno di assimilazione debba essere dovuto a reazione endotermica. Basandosi su questo semplice mezzo introdotto nella chimica biologica l'A. combatte alcuni schemi di reazioni già molto discussi. F con alcune considerazioni d'indole teoretica, riportandosi al concetto della simbiosi, l'A. chiude il suo lavoro, indicando le difficoltà che s'incontrano nello studio di simili fenomeni e le cause di tali difficoltà.

Va data lode all' $A$. per avere affrontato un argomento di così grande importanza, nulla trascurando di tutto quanto è stato scritto da illustri scienziati, alle cui fonti l'A. ha ricorso.

E. Manasse.

\section{Dizionagio tecnico in ouattro lingub, \\ (Italiano, Tedesco, Franoese, Ingless)}

Ing. E. WEBBER.

(Un volume - Hoepli, editore. Milano 1902 ).

E la seconda edizione del dizionario tecnico in quattro lingue, di cui già si occupò questo Giornale nel tomo IX, 1899 . pag. 224-225. La prima edizione fu accolta con grande favore poichè colmava una molto lamentata lacuna: toccherà a questa seconda ugual sorte, anzi migliore, poichè si presenta completamente riveduta ed accresciuta di ben 2000 termini tecnici.

M. A.

\section{PERTES D' ÉNERGIE DANS LES DIÉLECTRIQUES.}

Dott. PAUL L. MERCANTON.

(lansanne 1902).

In questa recentissima pubblicazione l'A. fa anzitutto un minuto ed accurato riassunto di quanto è stato fatto fin oggi sia dal lato elettrotecnico sia da quello teorico sull' importante argomento della dissipazione di energia elettrica nei coibenti. Aggiunge poi il contributo di numerose sue esperienze ese- 
guite negli anni 1900-1901, specialmente su miscugli dielettrici, perfezionando il metodo di ricerche giă adottato da F. Beaulard ${ }^{1}$ ).

F. Maccarrone.

\section{RIVISTA}

Journal de Physique. T. 10, serie, $3^{\text {n. }}$. Agosto a Dicembre, 1901.

Crémrgu V. Convezione elettrica e correnti aperte (pp. 453. 471). - Le conclusioni a cui giunge l'A. sono le seguenti :

Un disco girante in condizioni tali da trascurare la carica elettrica comunicatagli, non dà, facendo variare questa carica, gli effetti $d$ ' induzione che darebbe una corrente $d i$ conduzione che trasportasse quantità di elettricità uguali $\theta$ sottoposte a variazioni dello stesso ordine.

Un disco, girante nelle condizioni suddette non produce gli effetti magnetici di una corrente di conduzione che trasporta la stessa quantità d' elettricità.

La convezione elettrica non produce effetti magnetici ed esistono correnti aperte.

Pellat H. Sopra un fenomeno di oscillazione elettrica (pp. 471-475). - L'A. descrive un esperieuza a prima vista assai sorprendente. Due condensatori di capacità molto differenti hanno le loro armature rispettivamente riunite e sul cammino delle comunicazioni è inserito un invertitore che permette di far comanicare l'armatura positiva del condensatore piccolo con quella negativa del grande e viceversa. Vicino al condensatore piccolo si trova uno spinterometro in cui scocca la scintilla al momento opportuno. Se si caricano $i$ condensatori in modo di avere soltanto la metà della differenza di potenzialo ohe corrisponde alla scarica, o anche un poco meno, e si invertono le comunicazioni, la scintilla scocca sempre. Notiamo che, se la scintilla non scoceasse, quando l'equilibrio fosse ristabilito, la differenza di potenziale sarebbe minore di quella che era prima dell' inversione poichè le armature del condeusatore grande si farebbero parzialmente scaricate.

Cosi una causa, che nello stato di equilibrio, diminuisce la differenza di potenziale, fa aumentare questa differenza durante la rottura dell' equilibrio.

1) Journal de Physiqque, 3. serie, t. 9, 1900. 